\title{
Road users rarely use explicit communication when interacting in today's traffic: implications for automated vehicles
}

\author{
Yee Mun Lee ${ }^{1}\left[\right.$ Ruth Madigan $^{1} \cdot$ Oscar Giles $^{1} \cdot$ Laura Garach-Morcillo $^{1,2} \cdot$ Gustav Markkula $^{1} \cdot$ Charles Fox $^{1}$. \\ Fanta Camara ${ }^{1}$. Markus Rothmueller ${ }^{1,4}$. Signe Alexandra Vendelbo-Larsen ${ }^{1,4}$. Pernille Holm Rasmussen ${ }^{1,4}$. \\ Andre Dietrich $^{3}$. Dimitris Nathanael $l^{5} \cdot$ Villy Portouli $^{5} \cdot$ Anna Schieben $^{6} \cdot$ Natasha Merat $^{1}$
}

Received: 8 March 2019 / Accepted: 28 May 2020 / Published online: 8 June 2020

(c) The Author(s) 2020

\begin{abstract}
To be successful, automated vehicles (AVs) need to be able to manoeuvre in mixed traffic in a way that will be accepted by road users, and maximises traffic safety and efficiency. A likely prerequisite for this success is for AVs to be able to communicate effectively with other road users in a complex traffic environment. The current study, conducted as part of the European project interACT, investigates the communication strategies used by drivers and pedestrians while crossing the road at six observed locations, across three European countries. In total, 701 road user interactions were observed and annotated, using an observation protocol developed for this purpose. The observation protocols identified 20 event categories, observed from the approaching vehicles/drivers and pedestrians. These included information about movement, looking behaviour, hand gestures, and signals used, as well as some demographic data. These observations illustrated that explicit communication techniques, such as honking, flashing headlights by drivers, or hand gestures by drivers and pedestrians, rarely occurred. This observation was consistent across sites. In addition, a follow-on questionnaire, administered to a sub-set of the observed pedestrians after crossing the road, found that when contemplating a crossing, pedestrians were more likely to use vehiclebased behaviour, rather than communication cues from the driver. Overall, the findings suggest that vehicle-based movement information such as yielding cues are more likely to be used by pedestrians while crossing the road, compared to explicit communication cues from drivers, although some cultural differences were observed. The implications of these findings are discussed with respect to design of suitable external interfaces and communication of intent by future automated vehicles.
\end{abstract}

Keywords External-HMI · Human machine interface $\cdot$ Automated vehicles · Communication and interaction · Road safety · Pedestrians

\section{Introduction}

Yee Mun Lee

y.m.lee@leeds.ac.uk

1 Institute for Transport Studies, University of Leeds, Leeds LS2 9JT, UK

2 Department of Civil Engineering, University of Granada, Granada, Spain

3 Chair of Ergonomics, Technical University of Munich, Munich, Germany

4 School of Architecture, Design and Planning, Aalborg University, Aalborg, Denmark

5 Institute of Communications and Computer Systems, National Technical University of Athens, Athens, Greece

6 German Aerospace Centre (DLR), Braunschweig, Germany
According to the Society for Automotive Engineers (SAE 2018), highly automated (Level 4) driving is "the driving mode-specific performance by an automated driving system of all aspects of the dynamic driving task, even if a human driver does not respond appropriately to a request to intervene". An example of such vehicles is the automated buses and shuttles mostly used for demonstration and research purposes in many cities around the world, where conventional vehicle controls such as steering wheel and pedals are removed. In these vehicles, a "safety driver" monitors the system in case of emergencies, or system limitations, and some form of joystick and/or button is used to control the vehicle, if and when required. These are usually podlike vehicles, which can carry around $12-15$ passengers, and 
travel at low speeds (less than $20 \mathrm{~km} / \mathrm{h}$ ) along pre-defined routes (Alessandrini et al. 2015). Examples of such vehicle demonstrations, normally used for first/last mile transport, include those deployed by the CityMobil2 (Alessandrini et al. 2015), GateWay (GateWay 2016), and NS WePods (WePods 2016) projects in Europe, or the Navya trials in Las Vegas (Navya 2017).

Due to complexities of the urban environment, including its varied infrastructure, there is a mix of traffic between automated and non-automated vehicles, as well as other road users such as pedestrians and cyclists. Conventional vehicles with Level 4 capability are not yet available on our roads, although Original Equipment Manufacturers (OEMs) and new players of automated vehicle technologies, such as Waymo, Google and Apple, are currently conducting trials in this context in different cities around the world. In addition, national and European projects such as HumanDrive (https://humandrive.co.uk/), and L3Pilot (https://www.13pil ot.eu/) are working with a number of vehicle manufacturers to conduct on-road trials of automated driving at SAE Levels 3 and 4 . However, in terms of human factors, the focus in these trials has purely been on the on-board users' experience of the system, rather than any interactions with external road users.

In recent years, the interest in how, and whether, these vehicles should communicate and interact with other road users in complex urban traffic (including other drivers, and vulnerable road users (VRUs) such as pedestrians and cyclists), has increased (see Madigan et al. 2019; Rasouli and Tsotsos 2018; Rothenbucher et al. 2016). However, currently, official consideration of the value or success of bilateral interaction and communication methods between AVs and other road users is at a very early stage, although recent efforts by OEMs suggest that there may be an appetite for providing some form of externally facing communication by the $\mathrm{AV}$, as evidenced by promotion of various prototypes and concepts in the general media (Lagström and Lundgren 2015).

Although there is currently a limited understanding of the effects of these designs on actual road-user behaviour, example prototypes have included different forms of lighting on vehicles to signify automation state (on/off) and movement intention (stopping/starting/decelerating), as well as use of light reflections on roads and pavements, to provide messages and information. Discussions on this topic have also begun by standardisation organisations such as the United Nations Economic Commission for Europe (UNECE), the Society of Automotive Engineers (SAE J3016 ${ }^{\mathrm{TM}}$ ) and the International Organisation for Standardisation (ISO, TC22/ SC39/WG8 2020). Finally, following early reports from road users that some form of external communication method for acknowledging detection and relaying movement information by AVs may be useful (e.g. Merat et al. 2018; Schieben et al. 2018), a number of current and recent research projects have begun to examine the value of different forms of externally facing interfaces (collectively termed external human machine interfaces or eHMIs) on pedestrians' crossing behaviour (e.g. Ackermann et al. 2018; Clamann et al. 2017; Fridman et al. 2017). Studies in this context, which attempt to investigate road users' reactions to, and impressions of, these eHMI have relied on a number of techniques, including use of Wizard of Oz studies, which provide a "fake" automated vehicle (e.g. Habibovic et al. 2016; Rothenbucher et al. 2016), or virtual reality/head mounted displays (e.g. Ackermann et al. 2018; De Clercq et al. 2018; Deb et al. 2018; Hudson et al. 2019). Some studies have found beneficial effects of the presence of an eHMI in helping pedestrians make safe crossing decisions (e.g. Dey et al. 2017) and increasing their perceived safety around AVs (e.g. Habibovic et al. 2016). However, it is important to ensure that the eHMI conveys the message it intended, as Lee et al. (2019) found that the same eHMI could potentially be communicating two very different messages. For instance, their study showed that a pulsing light-based cue combined with a fast auditory sound cue was rated as the best signal for conveying two different messages, 'I am giving way' and 'I will start moving'. Other studies have failed to find any improvements in road users' comprehension of AV intentions with the inclusion of eHMI and have suggested that road users focus more on the movement patterns and speed of the vehicle to make their decisions (e.g. Clamann et al. 2017). To date, the focus of these studies has been on the factors influencing pedestrian and other road users' crossing decisions, and there have been no explorations of critical conflict scenarios.

\subsection{Observations of current traffic interactions}

In addition to studying road users' interactions with fabricated AVs, one area of research which may be beneficial for providing information in this field is observation of road user behaviour in current settings, to understand what cues pedestrians and car drivers use for successful interaction and communication in a mixed traffic environment.

Road user interactions have been studied by a range of research disciplines. These include studies from a traffic conflict and safety perspective, and investigations based on communication- and linguistics-based disciplines (see Markkula et al. 2020 for summary). As early as the 1960s/1970s, Goffman (1971/2010) was using a sociological perspective to identify how humans moving as "mobile shells" or "vehicular units" do not move irrespective of other such units, but are sensitive to, and recognise them, coordinating and adjusting their movements, to avoid collision and mutual obstruction. His work highlights how road users are repeatedly faced with challenges around how they can make their actions recognisable by others, and 
how they can establish, and maintain, mutual orientation with others, to engage in joint coordination of their mobile actions (Haddington and Rauniomaa 2014). Goffman identified how interaction rituals develop, whereby rules for behaviour are often governed by the frame through which participants interpret, and define, the situation at hand. In traffic scenarios, this interpretation is often influenced by road infrastructure, traffic rules, cultural expectations, etc. (Markkula et al. 2020). This type of work provides a strong theoretical framework for understanding the, often subtle, manner in which road users coordinate their movements, based on slight changes in trajectory, or informal communication chains, and how these movement patterns may differ across different locations/cultures.

Previous work regarding interaction of pedestrians in present road settings suggests that different forms of non-verbal interaction (such as eye contact, hand and/or head movements, or vehicle-based signals) are used. This form of communication is thought to be used by drivers to confirm to vulnerable road users (VRUs) that they are noticed, or given priority, in an interaction situation, i.e. a situation where the behaviour of at least two road users can be interpreted as being influenced by the possibility that they are both intending to occupy the same region of space at the same time in the near future (Markkula et al. 2020). This form of communication is also thought to increase compliance with traffic instructions and rules (Hamlet, Axelrod and Kuerschner 1984; Kleinke 1977). Such findings are used, for instance, by policy makers, such as the US Department of Transportation, to recommend that pedestrians 'make eye contact with drivers as they approach you to make sure you are seen' (California Department of Motor Vehicles 2015) and 'Make eye contact with the drivers of approaching vehicles whenever possible.' (Arizona Department of Transportation 2018) and also 'Make eye contact with drivers before you cross the street' (Alberta Transportation 2013).

Thus far, research studies attempting to understand how drivers and pedestrians communicate in current urban settings have delivered mixed results, with some studies suggesting that eye contact does play a role (Guéguen et al. 2015; Walker 2007; Sucha 2014), where others report that eye contact does not play a major role (Dey and Terken 2017), and that sometimes the presence of drivers is not even perceived (Risto et al. 2017; Sucha et al. 2017; Straub and Schaefer 2018). Explicit communication is also reported to be 'rare to non-existent' (Dey and Terken 2017; Rasouli et al. 2018), whereas the dynamic movement and behaviour of vehicles, such as speed changes (Várhelyi 1998), inching forward at intersections (Wang et al. 2014) and stopping behaviour (Dey and Terken 2017) are reported as more important cues.
Currently, over a third of globally reported traffic-related deaths and injuries occur between pedestrians and vehicles (Crandall et al. 2002; World Health Organisation 2013). A number of factors contribute to these figures, including the fact that pedestrians can determine their own course within the urban space, as they think best (Jian et al. 2005). Such flexibility also causes pedestrians to be one of the most unpredictable groups of road users, who cannot be effectively controlled by regulations (e.g. Jian et al. 2005; Cambon de Lavalette et al. 2009; Ward et al. 1994). Therefore, as the move towards higher levels of automation in vehicles increases, and the driver's responsibility for the control and manoeuvring of the vehicle is removed, it is reasonable to imagine that other forms of communication must be provided by the $\mathrm{AV}$, to replace the driver's role in this context. The hope is that this will ensure the same level, if not better, compliance with road rules and regulations, by these new forms of transport, ideally leading to higher trust and acceptance of AVs, and assisting in successful traffic flow between all actors in a mixed urban environment of the future, without compromising road safety.

As well as understanding how generic, and internationally agreed, rules of interactions between road users might inform AV behaviour, the role of 'social norms' in this context is also important (Goffman 2010; Rasouli and Tsotsos 2018). This includes, for example, understanding whether pedestrians from a particular region are more likely to use hand gesture as a form of communication while crossing the road, or whether the approaching car always yields to the pedestrians in certain regions, more than others. These are the informal rules which play a vital role in road users' interactions, including the ability to predict intention and behaviour (Evans and Norman 1998; Farber 2016; Lee and Sheppard 2016; Wilde 1980). Social norms are thought to differ from region to region, and even for different scenarios, and use cases (Straub and Schaefer 2018), which makes their adoption by $\mathrm{AVs}$ challenging. In addition, these norms may impact on road users' preferences about how an AV communicates with them, e.g. through auditory or visual cues (see Merat et al. 2018). Conte et al. (1998) mentioned that norms could make social behaviour more predictable. Therefore, it can be argued that conforming to social norms may increase an AV's safety, efficiency and acceptance on the road.

\subsection{Current study}

To further understand how pedestrians interact with vehicles in a mixed traffic setting, and establish whether social norms influence this behaviour, the current study used a newly developed observational protocol, and a follow-on on-site questionnaire, applied at six observed locations, in three European cities (Leeds, Munich and Athens). It should be noted that the focus of this study was on identifying 
communication patterns which may be possible to replicate or enact, using technology available to automated vehicles, and thus the focus was on easily identifiable explicit and implicit cues. The study aimed to explore a number of issues: (1) Do approaching vehicles provide explicit communication messages? (2) What types of vehicle movement behaviours are used to convey messages? (3) What movements do pedestrians make on the approach to a crossing point and during a crossing? The on-site questionnaire provided a further method for exploration of the use of vehicle- and driver-based information for pedestrians' crossing decisions, along with the manner in which pedestrians themselves believer they convey their crossing intentions.

\section{Method}

\subsection{Observation sites}

Six different observation sites were chosen across the three countries (see Fig. 1). These sites were chosen following a workshop between interACT project partners, which included the selection of suitable use cases for investigation (Wilbrink et al. 2017). Sites were chosen based on the criteria that communication would occur between road users, and that it was an urban area with a designated speed limit of $50 \mathrm{~km} / \mathrm{h}$. A separate set of studies have explored the types of communication occurring between pedestrians and vehicles in low-speed areas (see Uttley et al. 2020 under review).

To maximise the number of interactions between pedestrians and vehicles, one of the "must have" use cases chosen was one which included the need for a vehicle to "react to crossing of non-motorised traffic participants at crossings without traffic lights' (Wilbrink et al. 2017). The final locations for each city were chosen due to regular and numerous crossing opportunities for pedestrians, in the presence of approaching vehicles, which provided opportunity for frequent interactions between vehicles and pedestrians. Table 1 provides more information about each location, such as the map coordinates, location descriptions, road users' established priority rules, as well as the road's posted speed limit. Particular effort was placed on ensuring similarities between the sites as much as possible, although, for practical reasons, such as availability of suitable locations for video-recordings (not reported here), some compromises had to be made. In particular, these locations allowed a clear observation of where pedestrians looked during road crossings, along with providing some information of the different ways in
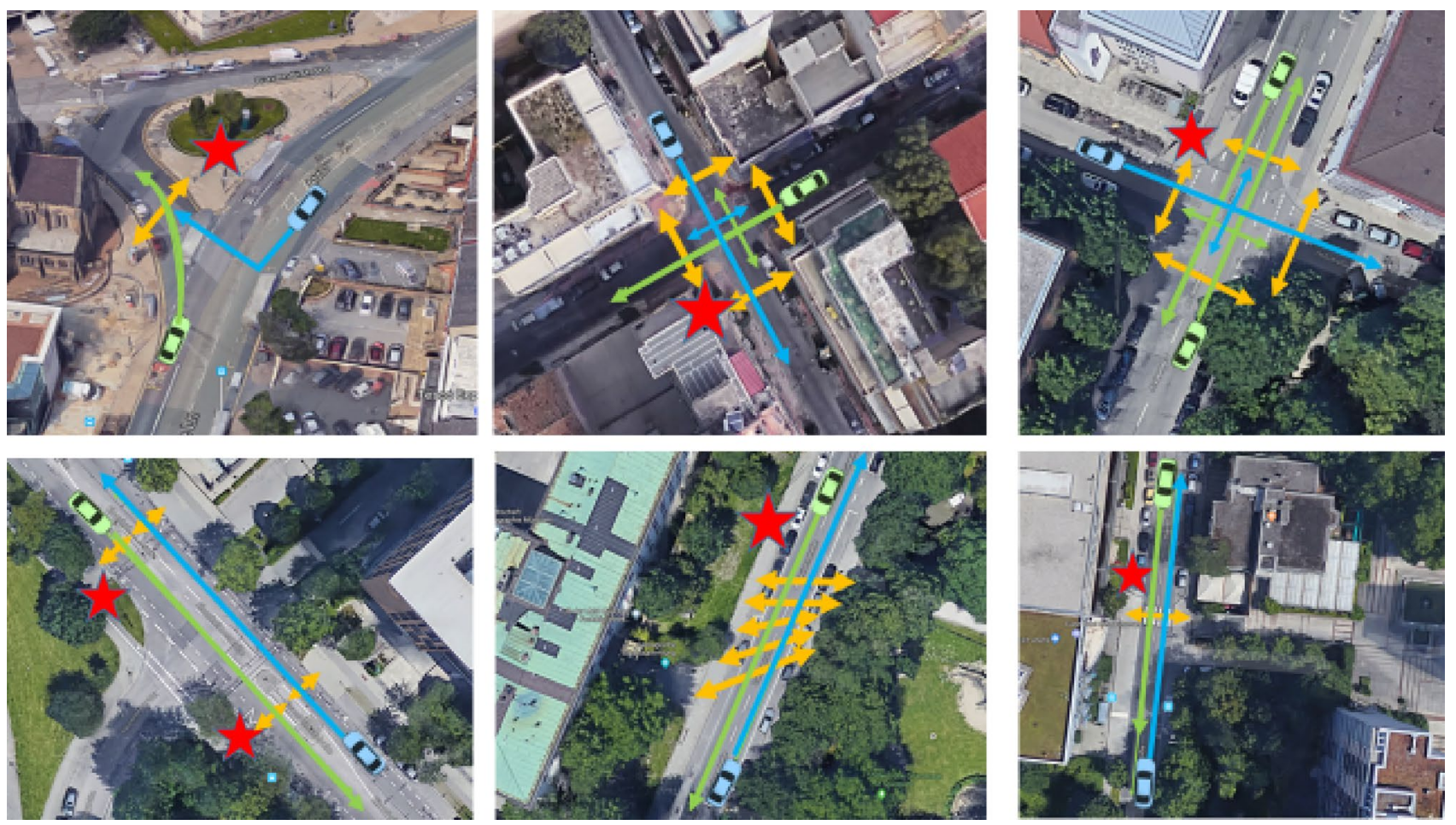

Fig. 1 Top left to right: The intersections at Leeds, Athens and Munich. Bottom left to right: Munich traffic island (two sections), Munich jaywalking, and Munich zebra crossing. Yellow arrows represent the location and direction of pedestrians' crossings. The blue and green lines represent where the approaching vehicles were coming from, and their respective arrows showing the directions they were travelling to. The red stars represent the location of a group of two observers, whereas the questionnaire administrator was mobile while administering the questionnaire (color figure online) 
Table 1 More information about the observation sites

\begin{tabular}{|c|c|c|c|c|}
\hline Sites & Map coordinate & Location description & Priority & Speed limit \\
\hline Leeds intersection & $53^{\circ} 483^{\prime} 26.3^{\prime \prime} \mathrm{N} 1^{\circ} 33^{\prime} 07.2^{\prime \prime} \mathrm{W}$ & University & Driver & $50 \mathrm{~km} / \mathrm{h}$ \\
\hline Athens intersection & $37^{\circ} 59^{\prime} 01.2^{\prime \prime} \mathrm{N} 23^{\circ} 43^{\prime} 17.9^{\prime \prime}$ & City centre & Driver & $50 \mathrm{~km} / \mathrm{h}$ \\
\hline Munich intersection & $48^{\circ} 09^{\prime} 02.8^{\prime \prime} \mathrm{N} 11^{\circ} 34^{\prime} 12.1^{\prime \prime} \mathrm{E}$ & $\begin{array}{l}\text { University, residential area, and } \\
\text { subway station }\end{array}$ & Driver & $\begin{array}{l}50 \mathrm{~km} / \mathrm{h} \text { north- } \\
\text { south, } 30 \mathrm{~km} / \mathrm{h} \\
\text { east-west }\end{array}$ \\
\hline Munich traffic island & $48^{\circ} 08^{\prime} 14.9^{\prime \prime} \mathrm{N} 11^{\circ} 31^{\prime} 19.6^{\prime \prime} \mathrm{E}$ & Offices and residential area & Driver & $50 \mathrm{~km} / \mathrm{h}$ \\
\hline Munich jaywalking & $48^{\circ} 08^{\prime} 50.9^{\prime \prime} \mathrm{N} 11^{\circ} 33^{\prime} 53.6^{\prime \prime} \mathrm{E}$ & University and museums & Driver & $50 \mathrm{~km} / \mathrm{h}$ \\
\hline Munich zebra crossing & $48^{\circ} 10^{\prime} 59.9^{\prime \prime} \mathrm{N} 11^{\circ} 32^{\prime} 12.6^{\prime \prime} \mathrm{E}$ & Shopping centre & Pedestrian & $50 \mathrm{~km} / \mathrm{h}$ \\
\hline
\end{tabular}

which drivers might communicate with other road users in un-signalised settings, where priority is unclear. Although this type of interaction might change across different infrastructure and driving environments, the inclusion of multiple sites allowed us to draw some conclusions on what typical interaction patterns might look like, and whether these were different across the three cities.

\subsection{Developing the observation protocol}

To provide a uniform method for recording behaviour at the chosen sites, an observation protocol was designed and converted into a digital application by the project team, which was then used for all locations. This application was usable on different electronic devices, available off-line, and also allowed the synchronisation of data between the video recordings (not reported here) and questionnaires (see also Dietrich and Ruenz 2019).

This pedestrian-vehicle observation protocol consisted of three main sections (see Fig. 2): The first section included 99 'event types', which captured observable behaviours of the pedestrians and vehicles, as they interacted with each other at each location, such as their looking behaviour, any observed hand gestures, signals and movements (see Camara et al. 2018). The second section of the application allowed recording of environment-based information, such as time of day and weather conditions. This section also included a recording of participants' demographic data (see Table 2).

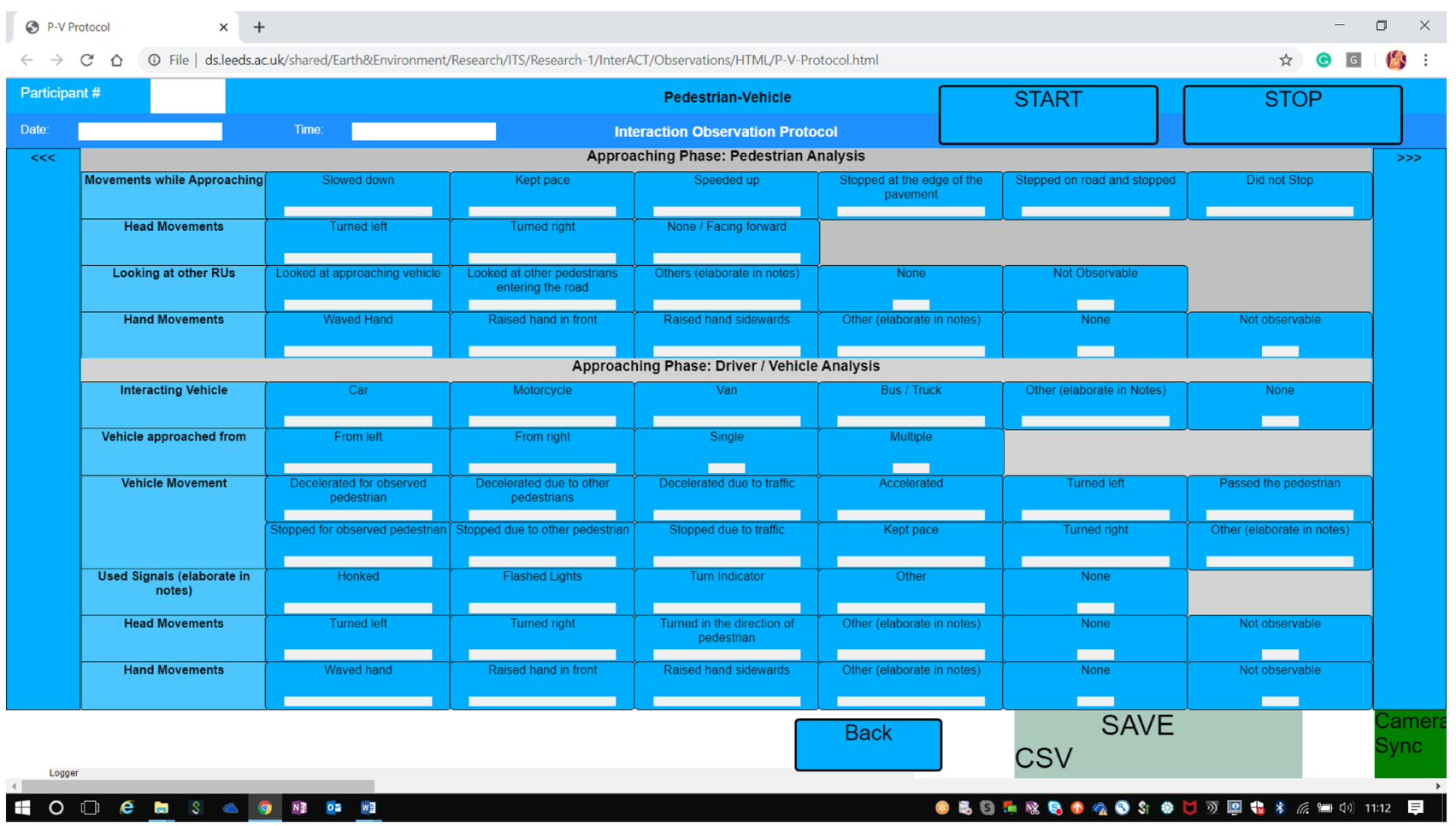

Fig. 2 A screen shot of the observation protocol application 
Table 2 Demographic data of pedestrians at each of the six observation sites

\begin{tabular}{|c|c|c|c|c|c|c|}
\hline Sites & Leeds intersection & Athens intersection & Munich intersection & Munich traffic island & Munich jaywalk & $\begin{array}{l}\text { Munich } \\
\text { zebra } \\
\text { crossing }\end{array}$ \\
\hline Total & $N=200$ & $N=211$ & $N=107$ & $N=87$ & $N=25$ & $N=71$ \\
\hline \multicolumn{7}{|l|}{ Individual/groups } \\
\hline$N$ available data & $(176)$ & (208) & $(104)$ & (86) & (24) & (71) \\
\hline Individual male & $30 \%(52)$ & $65 \%(135)$ & $36 \%(37)$ & $29 \%(25)$ & $50 \%(12)$ & $32 \%(23)$ \\
\hline Individual female & $36 \%(63)$ & $12 \%(25)$ & $42 \%(44)$ & $42 \%(36)$ & $17 \%(4)$ & $45 \%(32)$ \\
\hline Group & $35 \%(61)$ & $23 \%(48)$ & $22 \%(23)$ & $29 \%(25)$ & $33 \%(8)$ & $23 \%(16)$ \\
\hline \multicolumn{7}{|l|}{ Estimated age } \\
\hline$N$ available data & (190) & $(214)$ & $(107)$ & $(87)$ & (26) & (71) \\
\hline Child $(<13$ years old) & 0 & $3 \%(6)$ & $4 \%(4)$ & $2 \%(2)$ & 0 & 0 \\
\hline $\begin{array}{l}\text { Teenager (13-18 years } \\
\text { old) }\end{array}$ & $2 \%(4)$ & $0(1)$ & $1 \%(1)$ & 0 & 0 & $1 \%(1)$ \\
\hline $\begin{array}{l}\text { Young Adult (18-30 years } \\
\text { old) }\end{array}$ & $83 \%(158)$ & $24 \%(52)$ & $55 \%(59)$ & $15 \%(13)$ & $77 \%(20)$ & $25 \%(18)$ \\
\hline $\begin{array}{l}\text { Mid age adult }(30- \\
60 \text { years old })\end{array}$ & $14 \%(26)$ & $57 \%(123)$ & $33 \%(35)$ & $60 \%(52)$ & $15 \%(4)$ & $39 \%(28)$ \\
\hline $\begin{array}{l}\text { Older adult ( }>60 \text { years } \\
\text { old) }\end{array}$ & $1 \%(2)$ & $15 \%(32)$ & $7 \%(8)$ & $23 \%(20)$ & $8 \%(2)$ & $34 \%(24)$ \\
\hline
\end{tabular}

The third section provided a schematic representation of the junction, which allowed observers to draw any further details of the interaction, not recorded on the app, such as the direction of movement of the observed pedestrian and vehicle (not reported here).

\subsection{Developing the post crossing questionnaire}

To understand what factors pedestrians use for their crossing decisions, and whether vehicle- and driver-based information is used in this decision-making process, a short, 15-item, questionnaire was devised by the project team. These 15 questions included (1) Where are you travelling from? (5-item multiple choice question, MCQ) (2) Where are you travelling to? (5-item MCQ) (3) How regularly do you use this crossing? (7-item MCQ) (4) How safe did you feel during that crossing? (4-item Likert scale from very unsafe to very safe) (5) Why? (6) What information from the vehicle, if any, did you use to decide it was safe to cross? (8-item MCQ) (7) What information from the driver did you use to decide it was safe to cross? (6-item MCQ) (8) Was there any other information you used to determine how safe it was to cross? (9) How long did you feel they were waiting to find a suitable crossing gap? (3-item Likert from longer than usual to shorter than usual) (10) Did the presence of other people affect your decision of when to cross? (11) If so, in what way? (12) How did you indicate their intention to cross the road? (13) Who do you think has priority in this situation? (14) Are you a car driver? and (15) the ARUBQ questionnaire (see Elliott and Baughan 2004).
Here, pedestrians were also asked to report if they use any form of gesture and body language to communicate their intentions when crossing the road. The questionnaire was developed in English and translated into German and Greek by the relevant project partners. Before final administration, each translation was then checked by an independent German- or Greek-speaking colleague at Leeds. Due to its length, only some sections of the questionnaire (questions $6,7,8,12$ ), regarding pedestrians' use of cues, are included here.

\subsection{Procedure}

Ethical approval for this study was granted by the University of Leeds Ethics Committee (AREA 17-010). The studies were conducted on weekdays, between November and December 2017, with the weather conditions mostly sunny, or overcast, at all locations. Rush-hour times were avoided as it became too difficult to capture the details of interactions between two specific individuals. The same procedure was adopted for each observation site, with three observers for each site sharing the task of completing the observation protocol, and follow-on questionnaire (Sect. 2.3). As shown in Fig. 1, the three researchers (two observers and one questionnaire administrator) were positioned in a group, at a location, which was far enough from the intersection to avoid influencing the behaviour of the road users, but close enough for observing and recording the interactions.

The observers' main task was to identify a potential crossing manoeuvre by a pedestrian and record their behaviour 
during this exercise. For each observation recorded, one of the observers was responsible for choosing a pedestrian to observe, and the other focussed on the driver. Once the observation team were ready to begin an observation, they selected the next pedestrian whose trajectory suggested that they would be crossing the road, where there was also a vehicle approaching the intersection that they may have to interact with. A recorded observation started when the pedestrian was approximately $2-5 \mathrm{~m}$ away from the road (approaching phase) and continued until the pedestrian had arrived on the other side of the road (crossing phase). When observing the pedestrian/driver, the researchers took turns to vocalise the event types observed, with this data captured using a voice recorder. Once the interaction was complete, both observers completed the digital observation protocol together. This procedure was practiced extensively in a series of pilot observations studies lasting around a week, before the main data collection process occurred. In addition to assisting the observers with their roles and responsibilities, and fully familiarising them with their task, these pilot studies allowed sharing of any knowledge across the team, and an update of the app, if required. Following these pilot studies, some categories/sections were altered. For example, the category "clearly made eye contact" was removed after initial piloting showed that this was too difficult to establish with certainty, and the order of items was changed to make the app easier for users to navigate, by clustering the pedestrian and driver behaviours together.

Once a particular pedestrian's behaviour had been noted using the observation protocol, the questionnaire administrator approached the pedestrian and asked if they would be willing to complete a short questionnaire about the crossing that they had just made at the Leeds, Athens and Munich intersections. If they responded positively, they were provided with a short verbal introduction to the study and asked to sign a consent form. The questionnaire administrator then read the questions aloud, and also presented the questions visually to each participant, noting their answers on the questionnaire document. This change to verbal format followed the pilot study, since participants found it difficult to read the questions on the side of the street, and the process was found to be more efficient when the questions were read aloud. For multiple choice questions, the administrator left a gap after each option which allowed participants to respond immediately, rather than having to memorise the list. Participants were not compensated for their time to respond to the questionnaires, which took approximately $10 \mathrm{~min}$ to complete.

\subsection{Participants}

Table 2 provides a breakdown of the demographic information for pedestrians in each of the observation sites. Pedestrians' age was estimated to be one of five categories (child, teenagers, young, middle-aged, or older adults). The percentages reported are based on the number of valid cases, excluding cases where this detail was not recorded-see Table 2. Table 3 provides an overview of the demographic data of the pedestrians who took part in the post crossing questionnaire, summarised for each city.

A total of 989 observations were made. However, not all observations ended up in an interaction between the pedestrian and a vehicle, and therefore 288 cases were excluded. Thus, there were a total of 701 interactions across the six sites, where a pedestrian and vehicle approached the shared space at about the same time.

\section{Results and discussion}

\subsection{Observation protocol}

A total of 99 predefined temporal "event types" were recorded on the application, to define interactions between the pedestrians and vehicles/drivers during the pedestrians' approaching and crossing phase (see Camara et al. 2018). To allow a more simplified analysis framework, these event types were further collapsed and categorised according to similarities in gesture. For example, original event types 'waved hand', 'raised hand sideward', and 'raised hand in front' were collapsed into a new category described as 'pedestrian's hand gestures'. This provided 20 event types, which were defined by the pedestrians' behaviour (movement, looking behaviour, hand gesture) as well as the vehicles'/drivers' behaviour (movement, signal used, and drivers' hand gestures) during the whole course of the crossing (combining the approaching and crossing phase).

The percentages of each event type observed were then calculated for each site (see Table 4). Since a particular observation could include more than one event type, these behaviours were not mutually exclusive, and the data presented in this table, therefore, adds up to more than $100 \%$.
Table 3 Demographic data of the pedestrians at each location for post-crossing questionnaire

\begin{tabular}{lllllc}
\hline & $N$ & $\%$ of males $(N)$ & $\%$ of females $(N)$ & Mean age (SD) & Age range \\
\hline Leeds & 67 & $42 \%(28)$ & $58 \%(38)$ & $22.36(9.90)$ & $16-77$ \\
Athens & 63 & $78 \%(49)$ & $22 \%(14)$ & $42.37(14.15)$ & $19-74$ \\
Munich & 14 & $36 \%(5)$ & $64 \%(9)$ & $43.21(23.26)$ & $20-92$ \\
\hline
\end{tabular}


Table 4 The percentage (\%) of event types observed at each observation site

\begin{tabular}{|c|c|c|c|c|c|c|}
\hline Sites & $\begin{array}{l}\text { Leeds } \\
\text { intersection } \\
\%(N)\end{array}$ & $\begin{array}{l}\text { Athens } \\
\text { intersection } \\
\%(N)\end{array}$ & $\begin{array}{l}\text { Munich } \\
\text { intersection } \\
\%(N)\end{array}$ & $\begin{array}{l}\text { Munich } \\
\text { traffic island } \\
\%(N)\end{array}$ & Munich jaywalk \% $(N)$ & $\begin{array}{l}\text { Munich zebra } \\
\text { crossing } \%(N)\end{array}$ \\
\hline \multicolumn{7}{|l|}{ Pedestrians' movement } \\
\hline$N$ available data & $(200)$ & $(211)$ & $(107)$ & $(87)$ & $(25)$ & $(71)$ \\
\hline Decelerated and/stopped & $85 \%(169)$ & $56 \%(118)$ & $78 \%(83)$ & $99 \%(86)$ & $96 \%(24)$ & $66 \%(47)$ \\
\hline Kept pace when crossing & $41 \%(82)$ & $84 \%(177)$ & $21 \%(22)$ & $1 \%(1)$ & 0 & $34 \%(24)$ \\
\hline Accelerated when crossing & $7 \%(13)$ & $9 \%(19)$ & $26 \%(28)$ & $26 \%(23)$ & $16 \%(4)$ & $17 \%(12)$ \\
\hline Did not stop when crossing & $39 \%(77)$ & $38 \%(80)$ & $3 \%(3)$ & $9 \%(8)$ & 0 & 0 \\
\hline Initiated crossing movement & $88 \%(176)$ & $97 \%(204)$ & $64 \%(69)$ & $41 \%(36)$ & $100 \%(25)$ & $96 \%(68)$ \\
\hline \multicolumn{7}{|l|}{ Pedestrians' looking behaviour } \\
\hline$N$ available data & $(196)$ & $(211)$ & $(107)$ & $(87)$ & $(25)$ & $(71)$ \\
\hline $\begin{array}{l}\text { Looked towards approaching vehicles/ } \\
\text { drivers }\end{array}$ & $88 \%(173)$ & $86 \%(182)$ & $88 \%(94)$ & $98 \%(85)$ & $80 \%(20)$ & $93 \%(66)$ \\
\hline Looked at other pedestrians & $1 \%(1)$ & $9 \%(18)$ & $4 \%(4)$ & 0 & $12 \%(3)$ & $1 \%(1)$ \\
\hline \multicolumn{7}{|l|}{ Pedestrians' hand gestures } \\
\hline$N$ available data & $(199)$ & $(211)$ & $(107)$ & $(87)$ & $(25)$ & $(71)$ \\
\hline Hand gestures & $4 \%(8)$ & $4 \%(9)$ & $4 \%(4)$ & $11 \%(9)$ & 0 & $13 \%(8)$ \\
\hline \multicolumn{7}{|l|}{ Vehicles' movement } \\
\hline$N$ available data & $(200)$ & $(211)$ & $(107)$ & $(87)$ & $(25)$ & $(71)$ \\
\hline Decelerated for observed pedestrians & $30 \%(59)$ & $17 \%(36)$ & $21 \%(23)$ & $11 \%(10)$ & 0 & $73 \%(52)$ \\
\hline Decelerated for other pedestrians & $10 \%(20)$ & $0.5 \%$ & $1 \%(1)$ & 0 & 0 & $1 \%(1)$ \\
\hline Decelerated for vehicle traffic & $58 \%(116)$ & $18 \%(38)$ & $10 \%(11)$ & $9 \%(8)$ & $4 \%(1)$ & $4 \%(3)$ \\
\hline Stopped for observed pedestrians & $7 \%(13)$ & $9 \%(20)$ & $21 \%(23)$ & $10 \%(9)$ & 0 & $55 \%(39)$ \\
\hline Stopped for other pedestrians & $2 \%(4)$ & $2 \%(5)$ & $2 \%(2)$ & $1 \%(1)$ & 0 & $1 \%(1)$ \\
\hline Stopped for vehicle traffic & $27 \%(53)$ & $20 \%(43)$ & $25 \%(27)$ & $3 \%(3)$ & 0 & 0 \\
\hline Kept pace & $22 \%(43)$ & $50 \%(105)$ & $38 \%(41)$ & $49 \%(43)$ & $80 \%(20)$ & $15 \%(11)$ \\
\hline Accelerated & $18 \%(35)$ & $3 \%(6)$ & $17 \%(18)$ & $6 \%(5)$ & $24 \%(6)$ & $7 \%(5)$ \\
\hline Passed the pedestrian & $76 \%(152)$ & $39 \%(82)$ & $50 \%(54)$ & $70 \%(61)$ & $92 \%(23)$ & $14 \%(10)$ \\
\hline \multicolumn{7}{|l|}{ Vehicle signals } \\
\hline$N$ available data & (196) & $(211)$ & $(107)$ & $(87)$ & $(25)$ & $(71)$ \\
\hline Honked & 0 & 0 & 0 & 0 & 0 & 0 \\
\hline Flashed light & $1 \%(1)$ & $0.5 \%$ & 0 & 0 & 0 & 0 \\
\hline \multicolumn{7}{|l|}{ Drivers' hand } \\
\hline$N$ available data & $(138)$ & $(168)$ & $(50)$ & $(53)$ & $(0)$ & $(66)$ \\
\hline Hand gestures & $2 \%(3)$ & $3 \%(5)$ & $10 \%(5)$ & $6 \%(3)$ & 0 & $5 \%(3)$ \\
\hline
\end{tabular}

The following sections summarise the main findings from the observations.

\subsubsection{Do approaching vehicles provide explicit communication messages?}

From the 701 interactions observed across the six different locations, the results showed that there was almost no explicit communication message provided to pedestrians by the vehicles observed (less than $1 \%$ of vehicles honked their horn, or flashed their lights). In addition, only about $4 \%$ of drivers used hand gestures to communicate with pedestrians. Therefore, in contrast to previously proposed hypotheses (e.g. Wilde 1980; Clay 1995; Sucha et al. 2017), this study suggests that, currently, drivers do not use explicitly observable forms of communication when interacting with pedestrians in these particular urban use cases. Of particular value here is the uniform results observed across the three countries. Of course, it is important to highlight that this absence of explicit messages was observed for the particular range of road crossing situations studied here, where the posted speed limit was around $50 \mathrm{~km} / \mathrm{h}$. This is in contrast to studies conducted at sites with lower speed limits (e.g. Schneemann and Gohl 2016). Therefore, further observation studies in different urban environments are needed to ensure these results are representative (Uttley et al. 2020, under review). 


\subsubsection{Movement behaviour of the approaching car}

When pedestrians had priority, such as at the zebra crossing in Munich, the approaching vehicles were more likely to decelerate (74\%) and stop (56\%) to allow the pedestrians to cross. A less than $100 \%$ adherence to the law by these drivers may be due to the, sometimes, unclear placement of pedestrians and possible unpredictability of their intention. However, as observed by Sucha et al. (2017), in the city of Olomouc, $36 \%$ of drivers failed to yield to pedestrians at zebra crossings, even when pedestrians were waiting with the intention to cross (Várhelyi 1998).

On the other hand, in situations where the drivers had priority, i.e. in the absence of zebra crossings, observations showed that a much lower number of vehicles stopped or decelerated, with varied behaviours observed across the different infrastructure. For example, no vehicle decelerated or stopped for pedestrians in the jaywalk location in Munich, but $22 \%$ of vehicles decelerated, and $23 \%$ stopped when pedestrians attempted to cross at the intersection in the same city. In addition, at these sites, drivers were more likely to decelerate (average of $25 \%$ across sites) than stop (average of $17 \%$ across sites) for pedestrians. This suggests that, in the future, when AVs are adjusting their manoeuvre or yielding for pedestrians, it is acceptable for them to simply decelerate, rather than come to a full stop.

\subsubsection{Pedestrians' movements}

On average, only about $6 \%$ of pedestrians were seen to use some sort of hand gesture when crossing the road. Most pedestrians were seen to look towards the approaching vehicles (89\%) when crossing. Since the remaining pedestrians did not use an explicit communicative gesture before crossing the road, identifying their intention to cross is clearly a problematic process for both drivers, and, arguably, future automated vehicles.

For all of the sites, most of the observed pedestrians decelerated and/or stopped before crossing the road (average of $80 \%$ across sites), but this was noted less often at the Munich zebra crossing (66\%) and Athens intersection (56\%). Around 39\% of pedestrians at the Leeds intersection and $38 \%$ of those in Athens continued in their path and did not stop before crossing the road.

In summary, these observation studies, from the 701 interactions across three European cities, seem to suggest that pedestrians and drivers do not, in fact, exhibit explicit communicative gestures to indicate intention when interacting with each other in a mixed traffic environment. To investigate this matter further, and establish whether pedestrians are aware of, or indeed believe they are using any obvious external cues from drivers and vehicles when attempting a road crossing, more information was sought from the observed pedestrians via a short questionnaire, administered immediately after their crossing was observed.

\subsection{Questionnaire study}

\subsubsection{Use of vehicle- and driver-based information}

Participants were provided with a series of options and asked to state what vehicle-based and driver-based information they used to make their crossing decision for the particular crossing they had just made. They were allowed to choose more than one option (see Figs. 3 and 4).

The number of 'yes' responses provided by pedestrians for each category in this section was divided by the number of options available. For instance, for vehicle-based
Fig. 3 Percentage of pedestrians who responded 'yes' for each vehicle-based information used, for each city

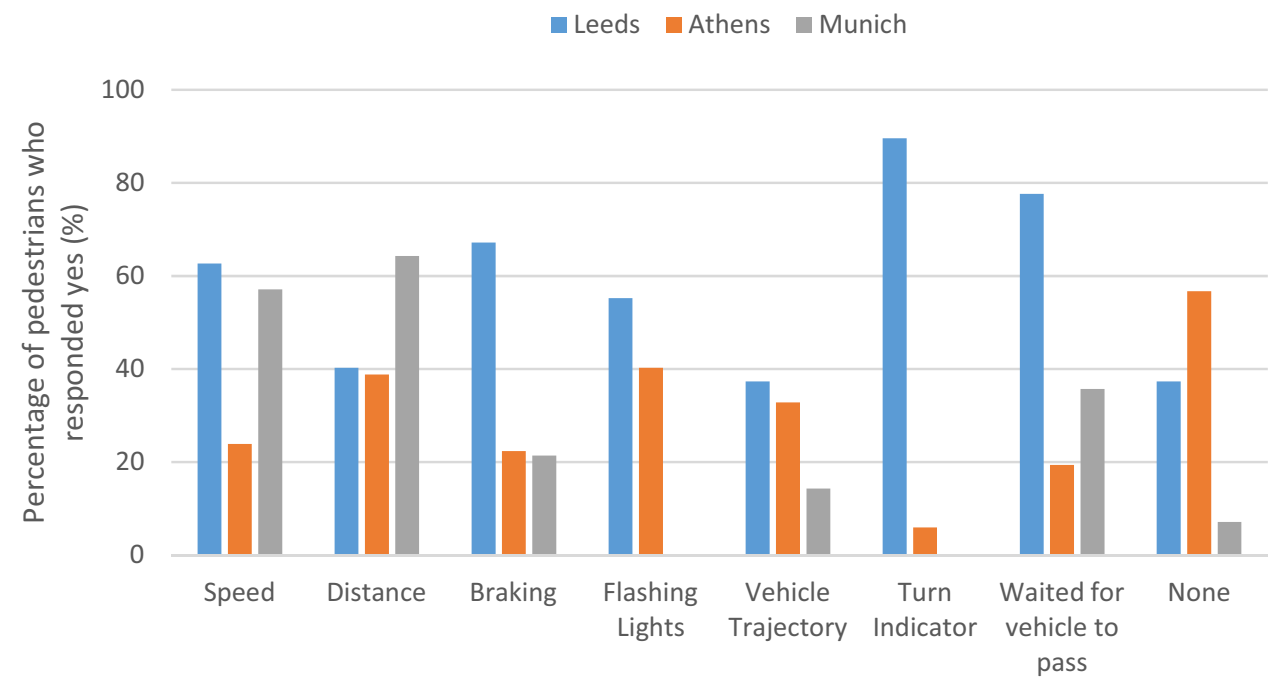

Vehicle-based Information 
Fig. 4 Percentage of pedestrians who responded 'yes' for each type of driver-based information used, for each city

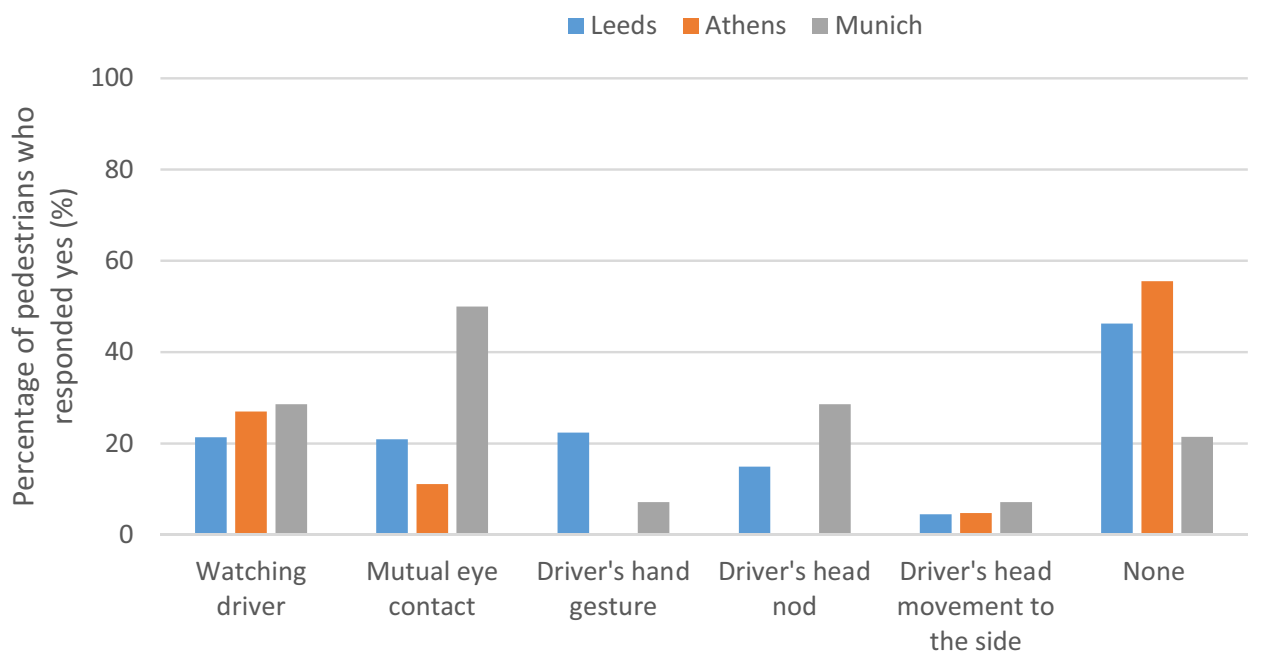

Driver-based Information information, if a pedestrian said that they used 'speed' and 'distance' only ( 2 of the 7 categories provided), they were taken to use $2 / 7=28.6 \%$ of the vehicle-based information provided.

Three paired-sample $t$ tests (one for each city) were conducted to investigate the proportion (\%) of vehiclebased and driver-based information used by pedestrians in each location. The paired-sample $t$ tests revealed that, compared to Munich, pedestrians in Leeds and Athens used vehicle-based information much more often than driver-based information, when deciding whether or not to cross the road (Leeds: $t(66)=6.15, p<0.001$; Athens: $t(63)=3.68, p<0.001$, Munich: $t(14)=0.489, p=0.633)$. However, this could be due to the smaller data set. About half of the pedestrians questioned in Leeds and Athens reported not using driver information at all while crossing at the intersection, whereas about $20 \%$ of the pedestrians questioned at the Munich intersection reported not using driver information.

When pedestrians were asked to provide details of any other information used when crossing ("Was there any other information you used to determine how safe it was to cross?'), additional responses were rare, but included auditory cues, i.e. 'listening', 'hearing', and 'sound of traffic' (Leeds, $N=3$ ), the use of nearby traffic lights to determine the manoeuvre of approaching vehicles (Leeds, $N=3$ ), and 'knowledge of normal behaviour at this crossing' (Leeds, $N=1$ ), 'common sense' (Leeds, $N=1$ ) and 'instinct' (Leeds, $N=1$ ). In Munich, only one pedestrian provided additional responses which was 'driving style' (Munich, $N=1$ ). Only two pedestrians provided further information in Athens, which included 'no, I just passed' and 'I don't remember'

\subsubsection{Indicating their intention to cross}

Pedestrians were also asked to indicate what kind of information they provided to show their intention to cross, for the crossing they had just made (see Fig. 5). As before, they were allowed to choose more than one option.

Over $50 \%$ of pedestrians did not report using any type of intention-signalling information to show their crossing intention, apart from 'stepping forward' which was reported by $70 \%$ of Munich pedestrians. It should be noted that although these results provided an indication of the information the pedestrians were conscious of considering, they do not necessarily capture any unconscious processing which may have occurred. In addition, there is a considerably lower number of data available for Munich intersections as compared to Leeds and Athens, and interpretation of this data should be done with some caution.

\section{General discussion and conclusions}

This study observed the movement and behaviour of pedestrians and drivers/vehicles at six designated locations in three European cities. The aim of the study was to establish if, and how, these actors communicate with one another when interacting in a mixed traffic environment, and especially when negotiating priority during use of the same road space. The ultimate aim of this study was to establish how such human-human interactions can be used to design suitable external interfaces for future automated vehicles, when the driver is no longer in charge of negotiating with pedestrians in such circumstances. Importantly, the focus of this paper has been on identifying patterns of human behaviour 
Fig. 5 Percentage of pedestrians who responded 'yes' for each intention information provided, for each location

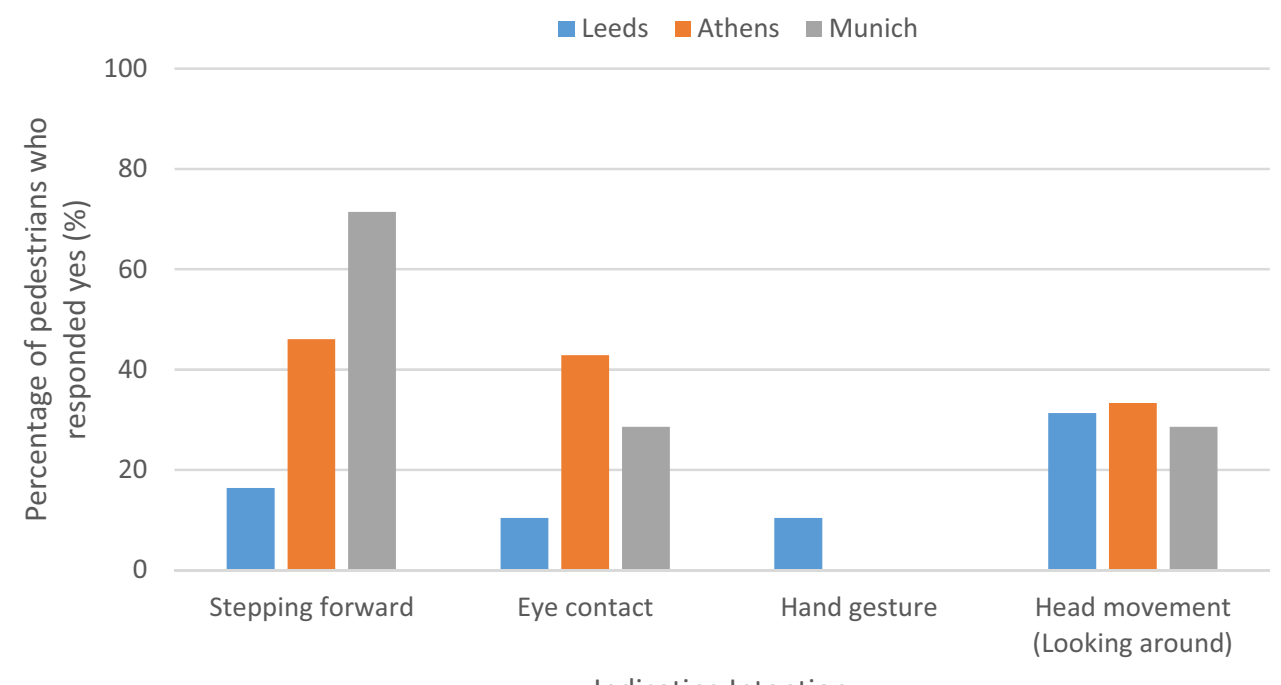

Indicating Intention which can be easily adapted for use in human-robot interactions, such as communication by future automated vehicles, where finer, and more discreet movements and gestures observed by previous sociological and linguistic studies may not be useful.

The results from the observation studies showed relatively consistent behaviour across the three cities, with pedestrians and drivers rarely using explicit body language to communicate with each other, relying instead on kinematic cues (such as distance, speed, braking). Follow-on questionnaires reported that pedestrians rely on factors such as vehicle speed, and distance, to decide on road crossing, relying less on driver communications such as eye contact, or head/hand movements. Only $27 \%$ of pedestrians reported using eye contact with the driver to signal that they intended to cross, while reciprocal eye contact by the driver, acknowledging pedestrian intention, was reported by $27 \%$ of pedestrians. To understand these findings in more detail, it would be useful if future studies used eye-tracking methods to determine whether eye contact has been established during interactions and how it affects communication and interactions.

Generally, in this study, we found that pedestrians reported using more vehicle-based, than driver-based, information to make a crossing decision. This finding was consistent across locations. These results support previous studies (Dey and Terken 2017; Risto et al. 2017; Sucha et al. 2017). However, there are a few important points to consider for interpreting these results. First, it is important to stress that these observations were conducted on roads with a $50 \mathrm{~km} / \mathrm{h}$ speed limit, and that more explicit communication may well occur between drivers and pedestrians sharing the same space on roads of lower speed limit (Schneemann and Gohl 2016; Uttley et al. 2020 under review). Such explicit communication may also be more prominent during deadlock scenarios, or encounters at short distances, where road users have to negotiate priority. Second, although quite rare, use of driver-based information was still reported. Therefore, future studies should consider the circumstances in which explicit communications between road users do actually occur. Third, although we have used this data to speculate what information may be needed from future automated vehicles, it is also important to address this question when pedestrians are interacting with actual AVs (e.g. Merat et al. 2018), since the shape and behaviour of these vehicles may be fundamentally different to that of current, manually driven vehicles. Finally, future studies should explore the impact of individual differences, such as road users' attitudes towards road interactions. For example, it can be argued that courtesy and altruism behaviour, or attitudes towards violations, may influence such interactions, with previous studies showing that these characteristics are linked to pedestrians' propensity to engage in risky and delinquent behaviours (see Elliott and Baughan 2004).

In terms of differences across the cities, our findings suggest that, compared to the other two cities, pedestrians in Athens were more likely to use eye contact to indicate crossing intention and were more likely to refrain from using any vehicle- or driver-based information. This highlights the potential need to consider the influence of different cultural and social norms when deploying AVs in different cities. It is also important to note that factors such as country-specific road design and infrastructure, traffic density, weather, etc. may have impacted on the interactions observed (see Madigan et al. 2019), and, therefore, any cross-cultural comparisons should be interpreted with caution.

Crucially, this exploratory observation study provides valuable insights, showing that, despite some country-specific infrastructural differences, across the range of locations, road users rarely used explicit communication to convey information about crossing intentions. This suggests that, 
in higher speed urban environments, road users will base their interactions on each other's movement patterns, indicating that there may be limited requirement for automated vehicles to adopt explicit communication solutions in these environments. Another interesting finding from our study was that drivers were more likely to decelerate $(16 \%)$, rather than come to a complete stop for pedestrians (11\%). Results in this context are somewhat conflicting, with Sucha et al. (2017) and Lee et al. (2019), suggesting that pedestrians are more likely to attempt crossing only when the vehicle has come to a full stop, with others showing that pedestrians often cross before the vehicle has come to a complete stop (e.g. Risto et al. 2017; Domeyer et al. 2019). At present, it seems reasonable to suggest that, to improve traffic flow, future AVs do not need to come to a complete stop, to aid pedestrians' crossing. Future studies should expand the scope provided here, to explore interactions between other road users such as vehicle-vehicle interactions and interactions with other vulnerable road users such as cyclists or older or younger age groups.

Acknowledgements This study was conducted as part of the interACT project, which received funding from the European Union's Horizon 2020 research and innovation programme, grant agreement No. 723395 .

Open Access This article is licensed under a Creative Commons Attribution 4.0 International License, which permits use, sharing, adaptation, distribution and reproduction in any medium or format, as long as you give appropriate credit to the original author(s) and the source, provide a link to the Creative Commons licence, and indicate if changes were made. The images or other third party material in this article are included in the article's Creative Commons licence, unless indicated otherwise in a credit line to the material. If material is not included in the article's Creative Commons licence and your intended use is not permitted by statutory regulation or exceeds the permitted use, you will need to obtain permission directly from the copyright holder. To view a copy of this licence, visit http://creativecommons.org/licenses/by/4.0/.

\section{References}

Ackermann C, Beggiato M, Bluhm L-F, Krems J (2018) Vehicle movement and its potential as implicit communication signal for pedestrians and automated vehicles. In: Proceedings of the 6th humanist conference, (June), 1-7. Retrieved from http://www. humanist-vce.eu/fileadmin/contributeurs/humanist/TheHague20 18/3-Ackerman.pdf

Alberta Transportation (2013) Traffic Safety in Alberta: 2013-2015 Communications Strategy. Retrieved from http://www.transporta tion.alberta.ca/Content/docType48/Production/OTSCommsSt rategy 13-15.pdf on 4/3/2019

Alessandrini A, Campagna A, Site PD, Filippi F, Persia L (2015) Automated vehicles and the rethinking of mobility and cities. Transp Res Proc 5:145-160. https://doi.org/10.1016/j.trpro.2015.01.002

Arizona Department of Transportation (2018) Arizona Driver License Manual and customer service guide. Retrived from https://www. azdot.gov/docs/default-source/mvd-forms-pubs/99-0117.pdf on 4/3/2019

California Department of Motor Vehicles (2015) California driver handbook-laws and rules of the road. Retrieved from https:// www.dmv.ca.gov/portal/dmv/detail/pubs/hdbk/right_of_way on $4 / 3 / 2019$

Camara F, Giles O, Madigan R, Rothmueller M, Rasmussen PH, Vendel-bo-Larsen A, Markkula G, Lee YM, Merat N, Fox CW (2018) Predicting pe-destrian road-crossing assertiveness for autonomous vehicle control. In: Proc. 21st IEEE international conference on intelligent transportation systems

Cambon de Lavalette B, Tijus C, Poitrenaud S, Leproux C, Bergeron J, Thouez JP (2009) Pedestrian crossing decision-making: a situational and behavioral approach. Saf Sci 47(9):1248-1253. https ://doi.org/10.1016/j.ssci.2009.03.016

Clamann M, Aubert M, Cummings ML (2017) Evaluation of vehicleto-pedestrian communication displays for autonomous vehicles. Tech, Rep

Clay D (1995) Driver attitude and attribution: implications for accident prevention. Ph.D. Dissertation, Cranfield University

Conte R, Castelfranchi C, Dignum FPM (1998) Autonomous norm acceptance. In: Müller J, Singh MP, Rao AS (eds) Intelligent agents $\mathrm{V}$, agent theories, architectures, and languages, proceedings 5th international workshop, ATAL'98, Paris, France, July 4-7, 1998. Springer, Berlin, pp 99-112. https://doi.org/10.1007/3540-49057-4_7

Crandall JR, Bhalla KS, Madeley NJ (2002) Designing road vehicles for pedestrian protection. BMJ Br Med J 324(7346): 1145

De Clercq K, Dietrich A, Núñez Velasco JP, De Winter J, Happee R (2018) External human-machine interfaces on automated vehicles : effects on pedestrian crossing decisions. Hum Factors 61(8):1353-1370

Deb S, Strawderman LJ, Carruth DW (2018) Investigating pedestrian suggestions for external features on fully autonomous vehicles: a virtual reality experiment. Transp Res Part F Traffic Psychol Behav 59:135-149. https://doi.org/10.1016/j.trf.2018.08.016

Deb S, Strawderman L, Carruth DW, DuBien J, Smith B, Garrison TM (2017) Development and validation of a questionnaire to assesspedestrian receptivity toward fully autonomous vehicles. Transp Res Part C 84:178-195

Dey D, Terken J (2017) Pedestrian interaction with vehicles: roles of explicit and implicit communication. In: International conference on automotive user interfaces and interactive vehicular applications, pp 109-113

Domeyer J, Dinparastdjadid A, Lee JD, Douglas G, Alsaid A, Price M (2019) Proxemics and kinesics in automated vehicle-pedestriancommunication: Representing ethnographic observations. Transp Res Rec 0361198119848413:1-12

Dietrich A, Ruenz J (2019) Observing traffic —utilizing a ground based LiDAR and observation protocols at a T-junction in Germany. In: Bagnara S, Tar-taglia R, Albolino S, Alexander T, Fujita Y (eds) Proceedings of the 20th congress of the international ergonomics association, IEA 2018. Advances in intelligent systems and computing, 823. Springer, Cham, 2019. https://doi.org/10.1007/9783-319-96074-6 56

Elliott MA, Baughan CJ (2004) Developing a self-report method for investigating adolescent road user behaviour. Transp Res Part F: Traffic Psychol Behav 7(6):373-393

Evans D, Norman P (1998) Understanding pedestrians' road crossing decisions: an application of the theory of planned behaviour. Health Educ Res 13(4):481-489

Färber B (2016) Communication and communication problems between autonomous vehicles and human drivers. In Autonomous driving: technical, legal and social aspects. Springer, Berlin, Heidelberg, pp 125-144. https://doi.org/10.1007/978-3-662-48847 $-8 \_7$ 
Fridman L, Mehler B, Xia L, Yang Y, Facusse LY, Reimer B (2017) To walk or not to walk: crowdsourced assessment of external vehicle-to-pedestrian displays. Retrieved from http://arxiv.org/ abs/1707.02698

GateWay (2016) Retrived from https://gateway-project.org.uk/ on $4 / 3 / 2019$

Goffman E (2010) Relations in public: microstudies of the public order. Transaction Press, New Brunswick (original work published 1971)

Guéguen N, Meineri S, Eyssartier C (2015) A pedestrian's stare and drivers' stopping behavior: a field experiment at the pedestrian crossing. Saf Sci 75:87-89. https://doi.org/10.1016/j. ssci.2015.01.018

Habibovic A, Andersson J, Nilsson M, Lundgren VM, Nilsson J (2016) Evaluating interactions with non-existing automated vehicles: three Wizard of $\mathrm{Oz}$ approaches. In: Intelligent vehicles symposium (IV), IEEE, pp 32-37

Haddington P, Rauniomaa M (2014) Interaction between road users: offering space in traffic. In: Space and culture, vol 17, no (2). SAGE Publications Inc., pp 176-90. https://doi. org/10.1177/1206331213508498

Hamlet CC, Axelrod S, Kuerschner S (1984) Eye contact as an antecedent to compliant behaviour. J Appl Behav Anal 17(4):553-557. https://doi.org/10.1901/jaba.1984.17-553

Hudson CR, Deb S, Carruth DW, McGinley J, Frey D (2019) Pedestrian perception of autonomous vehicles with external interacting features. AHFE. AISC 781:33-39. https://doi.org/10.1007/978-3319-94334-3 5

ISO, WG8/TC22 (2020) International Organization for Standardization Working Group 8

Jian L, Lizhong Y, Daoliang Z (2005) Simulation of bi-direction pedestrian movement in corridor. Phys Stat Mech Appl 354:619-628

Kleinke CL (1977) Compliance to requests made by gazing and touching experimenters in field settings. J Exp Soc Psychol 13(3):218 223. https://doi.org/10.1016/0022-1031(77)90044-0

Lagström T, Lundgren VM (2015) AVIP-Autonomous vehicles' ínteraction with pedestrians. Retrieved from http://publications.lib. chalmers.se/records/fulltext/238401/238401.pdf

Lee YM, Sheppard E (2016) The effect of motion and signalling on drivers' ability to predict intentions of other road users. Accid Anal Prev. https://doi.org/10.1016/j.aap.2016.07.011

Lee YM, Uttley J, Solernou A, Giles O, Markkula G, Romano R, Merat N (2019) Investigating pedestrians' crossing behaviour during car deceleration using wireless head mounted display: an application towards the evaluation of eHMI of automated vehicles. New Mexico, Driving Assessment

Madigan R, Nordhoff S, Fox C, Ezzati Amini R, Louw T, Wilbrink M, Schieben A, Merat N (2019) Understanding interactions between automated road transport systems and other road users: a video analysis. Transp Res Part F Traffic Psychol Behav 66:196-213

Markkula G, Madigan R, Nathanael D, Portouli E, Lee YM, Dietrich A, Billington J, Schieben A, Merat N (2020) Defining interactions: a conceptual framework for understanding interactive behaviour in human and automated road traffic. Theor Issues Ergon Sci (TTIE). https://doi.org/10.1080/1463922X.2020.1736686

Merat N, Louw T, Madigan R, Wilbrink M, Schieben A (2018) What externally presented information do VRUs require when interacting with fully Automated Road Transport Systems in shared space? Accid Anal Prev 118(April):244-252. https://doi. org/10.1016/j.aap.2018.03.018

Navya (2017) Navya and Keolis lauch the first autonomous, driverless and electric public transit vehicle to operate in US. Retrieved from https://navya.tech/en/navya-and-keolis-lauch-the-first-autonomusdriverless-and-electric-public-transit-vehicle-to-operate-in-u-s-2/ on $04 / 03 / 2019$
Rasouli A, Tsotsos JK (2018) Joint attention in driver-pedestrian interaction: from theory to practice, pp 1-111. Retrieved from http:// arxiv.org/abs/1802.02522

Rasouli A, Kotseruba I, Tsotsos JK (2018) Understanding pedestrian behavior in complex traffic scenes. IEEE Trans Intell Veh 3(1):6170. https://doi.org/10.1109/tiv.2017.2788193

Risto M, Emmenegger C, Vinkhuyzen E, Cefkin M, Hollan J (2017) Human-vehicle interfaces: the power of vehicle movement gestures in human road user coordination. In: Ninth international driving symposium on human factors in driver assessment, $p p$ 186-192

Rothenbucher D, Li J, Sirkin D, Mok B, Ju W (2016) Ghost driver: a field study investigating the interaction between pedestrians and driverless vehicles. In: 25th IEEE international symposium on robot and human interactive communication, RO-MAN 2016, pp 795-802. https://doi.org/10.1109/ROMAN.2016.7745210

SAE J3016 (2018) Taxonomy and definitions for terms related to onroad motor vehicle automated driving systems. https://www.sae. org/standards/content/j3016_201401/. Accessed 28 Feb 2019

Schieben A, Wilbrink M, Kettwich C, Madigan R, Louw T, Merat N (2018) Designing the interaction of automated vehicles with other traffic participants: design considerations based on human needs and expectations. Cognit Technol Work. ISSN 1435-5558. https ://doi.org/10.1007/s10111-018-0521-z

Schneemann F, Gohl I (2016) Analyzing driver-pedestrian interaction at crosswalks: a contribution to autonomous driving in urban environments. In: IEEE intelligent vehicles symposium (IV), Gothenburg, Sweden, June 19-22, 2016

Straub ER, Schaefer KE (2018) It takes two to Tango: automated vehicles and human beings do the dance of driving-four social considerations for policy. Transp Res Part A Policy Pract. https://doi. org/10.1016/j.tra.2018.03.005

Sucha M (2014) Road users' strategies and communication: driverpedestrian interaction. In: Transport research arena (TRA) 2014 proceedings. transport research arena (TRA) 5th conference: transport solutions from research to deployment. Paris, France

Sucha M, Dostal D, Risser R (2017) Pedestrian-driver communication and decision strategies at marked crossings. Accid Anal Prev 102:41-50. https://doi.org/10.1016/j.aap.2017.02.018

Uttley J, Lee YM, Madigan R, Merat N (2020) Investigating Road user interactions in a shared space setting: understanding what means of communication are needed for future automated vehicles. Transp Res Part F 72:32-46. https://doi.org/10.1016/j. trf.2020.05.004

Várhelyi A (1998) Drivers' speed behaviour at a zebra crossing: a case study. Accid Anal Prev 30(6):731-743. https://doi.org/10.1016/ S0001-4575(98)00026-8

Walker I (2007) Drivers overtaking bicyclists: objective data on the effects of riding position, helmet use, vehicle type and apparent gender. Accid Anal Prev 39(2):417-425. https://doi.org/10.1016/j. aap.2006.08.010

Wang C, Gu J, Terken J, Hu J (2014) Driver-to-driver communication on the highway: what drivers want. Ambient intelligence. In: Aarts E, de Ruyter B, Markopoulos P, van Loenen E, Schouten R, Wichert B, Terken J, Van Kranenburg R, Den Ouden E, O'Hare G, (eds) Springer International, Amsterdam, pp 315-327. https:// doi.org/10.1007/978-3-319-14112-1_25

Ward H, Cave J, Morrison A, Allsop R, Evans A (1994) Pedestrian activity and accident risk. Foundation for Road Safety Research, Basingstoke

WePod (2016) WePod-autonomous driverless vehicles. Retrived from http://www.smartcityembassy.nl/initiative/wepod-driverless-vehic les/ on $4 / 3 / 2019$

Wilbrink M, Schieben AM, Markowski R, Weber F, Gehb T, Ruenz J, Fabio T, Kaup M, Willrodt J, Portouli V, Merat N, Madigan R, Markkula G, Romano R, Fox C, Althoff M, Sontges S, Dietrich 
A (2017) interACT D1.1. Definition of interACT use cases and scenarios. https://www.interactroadautomation.eu/projects-deliv erables/

Wilde G (1980) Immediate and delayed social interaction in road user behaviour. Appl Psychol 29(4):439-460

World Health Organisation (2013) Global Status Report on Road Safety 2013: supporting a decade of action. World Health Organisation (WHO), Geneva
Publisher's Note Springer Nature remains neutral with regard to jurisdictional claims in published maps and institutional affiliations. 